\title{
Eosinophilia with Granulomatosis and Polyangiitis in a 57-Year-Old Man with Worsening Muscle Pain
}

Lauren K. King, MBBS, MSc; Julie Wright, MD; Christian Pagnoux, MD, MPH; Janice L. Kwan, MD, MPH

\section{About the Author}

Lauren King is a PGY2 Internal Medicine Resident at the University of Toronto. Julie Wright is a PGY3 Internal Medicine Resident at the University of Toronto. Christian Pagnoux is a Staff Rheumatologist at Mount Sinai Hospital in Toronto. Janice Kwan is a Staff General Internist at Mount Sinai Hospital in Toronto. Correspondence may be directed to janice.kwan@utoronto.ca.

\begin{abstract}
Eosinophilia with granulomatosis and polyangiitis (EGPA, previously called Churg-Strauss syndrome or allergic granulomatosis and angiitis) is an anti-neutrophil cytoplasmic antibody (ANCA)-associated vasculitis affecting small-sized vessels, which typically occurs in patients with a history of allergic rhinitis or asthma. The most serious cases arise from the involvement of other organ systems, notably the cardiovascular system. Importantly, patients may first exhibit non-specific manifestations such as malaise, fever, anorexia, and weight loss. This variable presentation and the potential for multi-organ involvement can lengthen time to diagnosis and delay treatment.

We describe a patient who presented with progressive myalgias on the background of recently diagnosed rhinosinusitis who was ultimately diagnosed with EGPA. This case is meant to alert general internists to the diagnostic challenges of EGPA.
\end{abstract}

\section{Résumé}

La granulomatose éosinophilique avec polyangéite (anciennement, le syndrome de Chrug et Strauss ou angéite allergique granulomateuse) est une vasculite liée à des anticorps anticytoplasme des polynucléaires neutrophiles (ANCA) qui touche les petits vaisseaux sanguins, apparaissant chez des personnes ayant des antécédents de rhinite allergique ou d'asthme. Les cas les plus graves surviennent quand la vascularite s'étend à d'autres systèmes organiques, plus particulièrement au système cardiovasculaire. Il est important de noter que les premières manifestations peuvent être d'ordre général, comme un malaise, de la fièvre, de l'anorexie et une perte de poids. En raison de ce tableau clinique variable et de la possibilité de l'atteinte multiorganique, le diagnostic peut être long à établir, ce qui risque de retarder le traitement. 


\section{Case}

A 57-year-old man presented to the emergency department with worsening muscle pain of the lower extremities, bilaterally. The patient had been well until approximately two weeks prior to presentation, when he noticed a deep ache in his thighs with activity and a precipitous decline in performance as goalie in his recreational hockey league ("I was letting in too many goals"). The pain became more severe, affecting his daily physical functioning; soon the discomfort prevented him from standing for more than 15 minutes at a time. He subsequently developed migratory swelling and pain, first affecting the right heel, then moving to the right wrist, the plantar aspect of his left foot, and the left knee. He began experiencing intermittent drenching night sweats and endorsed a recent weight loss of about $6 \mathrm{~kg}$. Finally, he developed bilateral shoulder and jaw pain that prompted him to seek medical attention by presenting to hospital.

His medical history included chronic rhinosinusitis with polyposis, diagnosed endoscopically 10 months earlier, for which he was taking inhaled budesonide and formeterol twice daily and mometasone furoate monohydrate intranasally as required. He did not have a history of asthma. He took no other medications.

The physical exam revealed vital signs, including temperature, that were within normal limits. Examination of his lower and upper limbs revealed no objective weakness and the neurological exam was normal. His gait was antalgic bilaterally and he was unable to hold his arms above his head secondary to pain. Respiratory exam was normal, musculoskeletal examination demonstrated no active joints, and there was no rash. He was admitted to hospital for further workup and management.

Initial blood work was significant for an elevated white blood cell count at $20.44 \times 10^{9} / \mathrm{L}$, with a differential revealing an eosinophil level of $9.61 \times 10^{9} / \mathrm{L}$. Electrolytes and creatinine were within normal limits. Testing of inflammatory markers revealed an erythrocyte sedimentation rate of $25 \mathrm{~mm} / \mathrm{hr}$ and a C-reactive protein of $82.9 \mathrm{mg} / \mathrm{L}$. Creatine phosphokinase (CPK) was mildly elevated at $371 \mathrm{IU} / \mathrm{L}$. Urinalysis showed 2+ blood. Chest radiograph was normal. Serology for parasitic infection and an autoimmune panel were pending.

His muscle pain was treated with naproxen $500 \mathrm{mg}$ orally twice daily. His symptoms improved and he was discharged the following day, with an outpatient rheumatology follow-up appointment in one week. Three days later, he returned to the emergency department with new- onset left foot numbness and interval development of a rash over his legs and scalp. His muscle pain returned and was significantly worse, affecting nearly his entire upper and lower limbs, with attenuation in the symptomatic relief provided by naproxen. On examination, he had decreased sensation to light touch affecting the left lower limb and evidence of new purpuric lesions on his palms, lower limbs, and dorsum of the feet. He also had blanchable papular lesions on his forehead. Musculoskeletal examination revealed tensosynovitis at the left second to fourth metatarsophalangeal joints. Testing for muscle power was limited secondary to pain. Repeat blood work demonstrated a rising CPK at 1,040. Testing for perinuclear (p)-anti-neutrophil cytoplasmic antibody (ANCA) was positive. Magnetic resonance imaging (MRI) of the lower limbs demonstrated diffuse edema of the musculature bilaterally consistent with myopathy. Nerve conduction and electromyography studies did not show a deficit. Echocardiogram and pulmonary function tests were normal. Chest computed tomography (CT) was subsequently performed and did not suggest active lung involvement. He was initiated on prednisone $60 \mathrm{mg}$ daily with rapid improvement of his myalgias. He was discharged from hospital with plans for close follow-up with a rheumatologist specialized in vasculitis.

\section{Discussion}

Eosinophilia with granulomatosis and polyangiitis (EGPA) is a vasculitis of the small-sized blood vessels associated with hyperesosinophilia and necrotizing granulomatous inflammation. ${ }^{1}$ It is a multisystem disorder typically occurring in patients with a history of allergic rhinitis or asthma. It has an incidence of $0.6-6.8$ per million per year. ${ }^{2}$ The mean age of diagnosis is 40 years, with no known gender predominance. ${ }^{3}$ Both vessel inflammation and eosinophilic proliferation are thought to play a role in organ damage, although the exact pathogenesis is not completely understood. ${ }^{4}$

Among the three ANCA-associated vasculidities (EGPA, granulomatosis with polyangiitis, and microscopic polyangiitis), EGPA is the least common. ${ }^{5}$ The most commonly involved organ is the lung, followed by ear, nose, and/or throat (ENT) involvement and skin; other frequently affected systems include the cardiovascular, gastrointestinal, renal, and central nervous systems. Nearly $40 \%$ of patients with EGPA have circulating ANCAs, predominantly directed against myeloperoxidase, with a perinuclear staining pattern (p-ANCA). ${ }^{6}$ Different subtypes of disease may exist with clinical characteristics that differ according to ANCA status ${ }^{7}$ : patients with ANCA positivity have been found to be more affected with renal disease, mononeuritis, purpura, and alveolar haemorrhage; cardiac involvement, lung infiltration, and systemic manifestations are more frequently seen in patients who are ANCA negative., ${ }^{6,8}$ 
The American College of Rheumatology has developed six criteria for the classification of EGPA: presence of asthma; eosinophilia $>10 \%$; mononeuropathy or polyneuropathy; pulmonary infiltrate; non-fixed paranasal sinus abnormality; and extravascular eosinophils on biopsy of a blood vessel. The presence of any four or more of the six criteria produces a sensitivity of $85 \%$ and a specificity of $99.7 \%$ for diagnosis. ${ }^{9}$ Importantly, these criteria were devised as a classification system to distinguish EGPA from other vasculitidies, rather than a formal checklist for the diagnosis of EGPA.

The presentation of EGPA is far from uniform. The initial phase can last years and generally involves allergic rhinitis, nasal polyposis and sinusitis, and/or asthma. ${ }^{2}$ This is followed by the occurrence of vasculitic manifestations, such as skin involvement and/or mononeuritis multiplex. Constitutional symptoms are also often present at diagnosis (40-75\%), including fever, weight loss, myalgias, and arthralgias. ${ }^{2}$ At diagnosis, $40-75 \%$ of patients have skin involvement, most frequently palpable purpura, but other lesions such as cutaneous nodules or papules with an urticarial appearance can be seen. ${ }^{2}$ Other organ systems are also frequently involved. Between $38-77 \%$ of patients can have parenchymal lung involvement. Gastrointestinal manifestations, which occur in $20-50 \%$ of patients, may include abdominal pain, vomiting, or diarrhea. ${ }^{10}$ Cardiac involvement, present in $27-47 \%$ of patients, includes cardiomyopathy and eosinophilic pericardial effusion and is of paramount importance to discern due to its heightened association with disease-related morbidity. ${ }^{4,11} \mathrm{Up}$ to $27 \%$ of patients may have renal involvement, mainly in the form of focal segmental or pauci-immune glomerulonephritis. ${ }^{12}$

The initial and cornerstone of treatment for EGPA is corticosteroids. For patients with evidence of systemic vasculitis, treatment is initiated with prednisone (or equivalent) at a dose of $1 \mathrm{mg} / \mathrm{kg}$ per day. Additional immunosuppressive agents are typically added in cases of refractory or severe disease, such as impending respiratory failure, cardiac involvement, glomerulonephritis, and/or severe motor neuropathy. Most patients with EGPA improve with glucocorticoid therapy alone. ${ }^{13}$ Once symptomatic remission has been achieved, the dosage of steroid is gradually tapered over $12-18$ months, as tolerated by the patient. However, a report of patients without poor prognosis factors (i.e., no cardiac, renal, or central nervous system involvement) indicated that almost $80 \%$ of those who achieved remission required long-term, low-dose glucocorticoid therapy because of lingering asthma or rhinitis. ${ }^{13}$

\section{Conclusion}

This was the case of a 57-year-old man with progressive myalgias affecting the upper and lower limbs, associated with weight loss and migratory tenosynovitis, and with a history of allergic rhinitis. Diagnosis only became apparent as the other manifestations of EGPA developed with eosinophilia, skin involvement, and neuropathy. This case highlights the challenges associated with diagnosing EGPA. Given the multi-system and heterogeneous nature of the disease, keeping the diagnosis on one's radar, recognizing its presenting features, and including it in the differential diagnosis is essential.

\section{References}

1. Samson M, Puechal X, Devilliers H, et al. Long-term outcomes of 118 patients with eosinophilic granulomatosis with polyangiitis (Churg-Strauss syndrome) enrolled in two prospective trials. J Autoimmun 2013;43:60-9.

2. Hoffman GS, Veyand CM, Langford CA, eds. Inflammatory Diseases of Blood Vessels. 2nd Ed. West Sussex: Wiley-Blackwell; 2012.

3. Conron M, Beynon HL. Churg-Strauss syndrome. Thorax 2000;55:870-7.

4. Mahr A, Moosig F, Neumann T, et al. Eosinophilic granulomatosis with polyangiitis (Churg-Strauss): evolutions in classification, etiopathogenesis, assessment and management. Curr Opin Rheumatol 2014;26:16-23.

5. Keogh KA, Specks U. Churg-Strauss syndrome. Semin Respir Crit Care Med 2006;27:148-57.

6. Pagnoux C, Guillevin L. Churg-Strauss syndrome: evidence for disease subtypes? Curr Opin Rheumatol 2010;22:21-8.

7. Pagnoux C, Guilpain P, Guillevin L. Churg-Strauss syndrome. Curr Opin Rheumatol 2007;19:25-32.

8. Sinico RA, Bottero P. Churg-Strauss angiitis. Best Pract Res Clin Rheumatol 2009;23:355-66.

9. Masai A, Hunder G, Lie J,. The American College of Rheumatolgoy 1990 criteria for the classification of Churg-Strauss syndrome (allergic granulomatosis and angiitis). Arthritis Rheum 1990;33:1094-100.

10. Pagnoux C, Mahr A, Cohen P. Presentation and outcome of gastrointestinal involvement in systemic necrotizing vasculitides: analysis of 62 patients with polyarteritis nodosa, microscopic polyangiitis, Wegener granulomatosis, Churg-Strauss syndrome, or rheumatoid arthritis-associated vasculitis. Medicine (Baltimore) 2005;84:115-28.

11. Langford C. Clinical features and diagnosis of small-vessel vasculitis. Cleve Clin J Med 2012;79Suppl 3:S3-7.

12. Sinico RA, Di Toma L, Maggiore U, et al. Renal involvement in ChurgStrauss syndrome. Am J Kidney Dis 2006;47:770-9.

13. Ribi C, Cohen P, Pagnoux C, et al. Treatment of Churg-Strauss syndrome without poor-prognosis factors: a multicenter, prospective, randomized, open-label study of seventy-two patients. Arthritis Rheum 2008;58:586-94. 